\title{
Constitution de la République de Costa Rica
}

du 7 novembre 1949 et ses révisions

\section{(2) OpenEdition}

\section{Journals}

Édition électronique

URL : http://journals.openedition.org/plc/844

DOI : $10.4000 /$ plc. 844

ISSN : 2117-5209

\section{Éditeur}

L'Harmattan

\section{Édition imprimée}

Date de publication : 1 janvier 2010

Pagination : 209-275

ISBN : 978-2-296-1141-3

ISSN : 1279-8657

\section{Référence électronique}

"Constitution de la République de Costa Rica », Pouvoirs dans la Caraïbe [En ligne], 16 | 2010, mis en ligne le 22 septembre 2011, consulté le 19 avril 2019. URL : http://journals.openedition.org/plc/844 ; DOI : $10.4000 /$ plc. 844

Ce document a été généré automatiquement le 19 avril 2019

(c) Pouvoirs dans la Caraïbe 


\section{Constitution de la République de Costa Rica ${ }^{1}$}

du 7 novembre 1949 et ses révisions

1 Nous, représentants du peuple de Costa Rica, librement élus députés à l'Assemblée Nationale Constituante, invoquant le nom de Dieu et réaffirmant notre foi en la démocratie, décrétons et adoptons la Constitution suivante :

\section{TITRE I}

LA REPUBLIQUE

Chapitre unique

4 Article 1. - Costa Rica est une République démocratique, libre et indépendante.

5 Article 2. - La souveraineté réside exclusivement dans la Nation.

6 Article 3. - Nul ne peut s'arroger la souveraineté ; celui qui le ferait commettrait un délit de trahison envers la Patrie.

7 Article 4. - Nulle personne ou réunion de personnes ne peut assurer la représentation du peuple, s'arroger ses droits ou faire des pétitions en son nom. Toute infraction à cet article serait une sédition.

8 Article 5. - Le territoire national est compris entre la mer des Caraïbes, l'Océan Pacifique et les Républiques de Nicaragua et de Panama.

9 Les frontières de la République sont celles qui ont été définies par le traité Canas-Jerez du 15 avril 1858, confirmé par l'arbitrage Cleveland du 22 mars 1888 en ce qui concerne le Nicaragua, et le Traité Echandi Montero-Fernandez Jaen du $1^{\text {er }}$ Mai 1941 en ce qui concerne Panama.

10 L'île de Coco, située dans l'Océan Pacifique, constitue une partie du territoire national.

11 Article 6. - L'Etat exerce une souveraineté complète et exclusive sur l'espace aérien de son territoire, sur ses eaux territoriales sur une distance de douze milles à partir d'une ligne 
de basse mer le long de ses côtes, sur son plateau continental et sur le socle insulaire, en accord avec les principes du droit international.

De plus, il exerce une juridiction spéciale sur les mers adjacentes à son territoire jusqu'à une distance de deux cents milles à partir de la ligne de basse mer, afin de protéger, conserver et exploiter en exclusivité toutes les ressources et richesses naturelles existantes dans les eaux, le sol et le sous-sol de ces zones, conformément aux principes précités.

(Réforme constitutionnelle, loi $n^{\circ} 5699$ du 5 juin 1975)

Article 7. - Les traités publics, les conventions internationales et les concordats dûment approuvés par l'Assemblée législative auront, à compter de leur promulgation ou à compter du jour qu'ils précisent, une autorité supérieure aux lois.

Les traités publics et les conventions internationales visant l'intégrité territoriale ou l'organisation politique du pays, requièrent l'approbation de l'Assemblée législative, par un vote à la majorité des trois-quarts de la totalité de ses membres, et les deux-tiers des membres d'une Assemblée Constituante, convoquée à cet effet.

(Réforme constitutionnelle, loi $n^{\circ} 4123$ du 31 mai 1968)

Article 8. - Les Etats étrangers pourront seulement acquérir sur le territoire de la République, sur la base de la réciprocité, les immeubles nécessaires à leurs représentations diplomatiques, sans préjudice de ce qui est prévu par les conventions internationales.

Article 9. - Le gouvernement de la République est populaire, représentatif, alternatif et responsable.

Il est exercé par trois pouvoirs distincts et indépendants : Législatif, Exécutif et Judiciaire.

Aucun de ces pouvoirs ne peut déléguer l'exercice des fonctions qui lui sont propres.

Un Tribunal Suprême Electoral, de même rang et jouissant de la même indépendance que les Pouvoirs de l'Etat, a à sa charge de façon exclusive et indépendante l'organisation, la direction et la surveillance des opérations relatives au vote, ainsi que, de plus, les autres fonctions que lui attribuent la Constitution et les lois.

(Réforme constitutionnelle, loi $n^{\circ} 5704$ du 5 juin 1975)

Article 10. - Relèvera d'une Chambre spécialisée de la Cour suprême de Justice de déclarer, à la majorité absolue de ses membres, l'inconstitutionnalité des normes de quelque nature et des actes soumis au droit public. Ne seront pas attaquables les actes juridictionnels du Pouvoir judiciaire, la déclaration de l'élection que prononce le Tribunal Suprême Electoral et les autres actes qui seront déterminés par la loi.

De plus, il en relèvera :

a) De résoudre les conflits de compétence entre les pouvoirs de l'Etat, incluant le Tribunal Suprême Electoral, ainsi que de plus, les entités ou organes indiqués par la loi.

b) D'être consulté sur les projets de réforme constitutionnelle, d'approbation des conventions et traités internationaux et autres projets de loi, selon ce qu'en dispose la loi.

(Réforme constitutionnelle, loi n $n^{\circ} 5701$ du 5 juillet 1975

et loi $n^{\circ} 7128$ du 18 août 1989)

Article 11. - Les fonctionnaires sont de simples dépositaires de l'autorité et ne peuvent s'arroger des pouvoirs que la loi ne leur accorde pas. Ils doivent prêter le serment 
d'observer et appliquer cette Constitution et les lois. La mise en cause de leur responsabilité pénale fait l'objet d'une action publique.

L'Administration Publique au sens large, sera soumise à une procédure d'évaluation des résultats et de reddition de comptes, avec la responsabilité personnelle conséquente à l'accomplissement de leurs devoirs pour les fonctionnaires. La loi indiquera les moyens pour que ce système de contrôle des résultats et de reddition des comptes couvre toutes les institutions publiques.

(Réforme constitutionnelle, loi nº 8003 du 8 juin 2000)

Article 12. - L'armée est interdite en tant qu'institution permanente.

La surveillance et la préservation de l'ordre public sont assurées par les forces de police nécessaires.

Les forces militaires pourront seulement être organisées en vertu d'un accord continental ou pour la défense nationale; dans les deux cas, elles seront toujours subordonnées au pouvoir civil ; nul ne pourra délibérer, ni faire de manifestations ou déclarations sous une forme individuelle ou collective.

\section{TITRE II}

\section{LES COSTARICAINS}

Chapitre unique

Article 13. - Sont costaricains par la naissance :

1) L'enfant de père et de mère costaricain né sur le territoire de la République ;

2) L'enfant de père ou de mère costaricain par la naissance, qui naît à l'étranger, et est inscrit comme tel à l'état civil, par la volonté de l'un de ses parents costaricains, pendant sa minorité, ou par sa propre volonté avant qu'il ait atteint vingt ans ;

3) L'enfant de parents étrangers né au Costa Rica qui est inscrit en tant que costaricain, par la volonté de ses parents pendant sa minorité, ou avant qu'il ait atteint vingt ans ;

4) Le petit enfant, de parents inconnus, trouvé à Costa Rica.

Article 14. - Sont costaricains par naturalisation :

1) Ceux qui ont acquis cette nationalité en vertu de lois antérieures.

2) Les nationaux des autres pays d'Amérique centrale, les Espagnols et Ibérico-Américains par naissance et qui possèdent une résidence officielle dans ce pays durant cinq années au minimum et accomplissent de plus les formalités requises par la loi.

3) Les Centraméricains, les Espagnols et les Ibérico-Américains qui ne sont pas costaricains par naissance et qui ayant une résidence officielle dans ce pays durant sept années au moins et qui accomplissent les formalités diverses requises par la loi.

4) La femme étrangère qui contracte un mariage avec un Costaricain et a perdu sa nationalité.

5) Les personnes étrangères qui en se mariant avec des Costaricains perdent leur nationalité ou qui après avoir été mariées durant deux ans à des Costaricains et résidé durant cette même période dans le pays, manifestent leur désir d'acquérir la nationalité costaricaine

(Réforme constitutionnelle, loi n 7879 du 27 mai 1999) 

démontrer qu'il détient un métier ou un moyen connu de vivre, qu'il sait parler, écrire et lire l'idiome espagnol, se soumettre à un examen de connaissance de l'histoire du pays et ses valeurs, promettre de résider sur le territoire national de manière régulière et jurer qu'il respectera l'ordre constitutionnel de la République. République, ou ailleurs, à condition d'être libre de toute responsabilité, et y revenir quand il veut. On ne pourra exiger des costaricains des formalités empêchant leur rentrée au pays. 
69 Article 23. - Le domicile et tout autre local privé des habitants de la République sont inviolables. Nonobstant qu'ils puissent être perquisitionnés sur ordre écrit du juge compétent, soit pour empêcher qu'un crime soit commis ou demeure impuni, soit pour éviter des dommages graves à des personnes ou à des biens, sous réserve des dispositions prévues par la loi.

Article 24. - Le droit à l'intimité, à la liberté et au secret des communications est garanti.

71 Les documents privés et les communications écrites, orales ou d'un quelconque autre type des habitants de la République sont inviolables. Cependant, la loi, dont l'approbation et la réforme requièrent le vote des deux-tiers des députés de l'Assemblée législative, précisera dans quels cas les Tribunaux ordonneront le séquestre, l'enregistrement ou l'examen des documents privés, lorsque c'est absolument indispensable pour éclaircir des affaires qui leur sont soumises.

72 La loi déterminera également dans quels cas les Tribunaux pourront ordonner qu'intervienne leur communication et préciser les délits pour lesquels l'enquête pourra autoriser l'usage de ce pouvoir exceptionnel et durant combien de temps. De la même manière, elle précisera les responsabilités et sanctions qu'encourent les fonctionnaires qui appliquent illégalement cette exception. Les décisions judiciaires protégeant cette règle doivent être raisonnables et pourront être exécutées immédiatement. Leur application et leur contrôle seront assurés sous la responsabilité, qui ne peut être déléguée, de l'autorité judiciaire.

73 La loi précisera les cas dans lesquels les fonctionnaires compétents du Ministère des Finances Publiques et de l'Inspection Générale de la République pourront contrôler les livres de comptabilité et leurs annexes à des fins fiscales et pour fiscaliser la correcte utilisation des fonds publics.

Une loi spéciale, approuvée par les deux-tiers des Députés, déterminera quels autres organes de l'Administration publique pourront contrôler les documents que cette loi précisera en relation avec l'accomplissement de leurs compétences, de réglementation et de surveillance pour le respect d'objectifs publics. De la même manière, elle indiquera dans quels cas procéder à cette révision.

N'auront aucun effet légal, la correspondance qui sera soustraite et l'information obtenue comme résultant de l'intervention illégale de cette communication.

(Réforme constitutionnelle, loi $n^{\circ} 7607$ du 29 mai 1996)

Article 25. - Les habitants de la République disposent du droit d'association à des fins licites. Nul ne peut être obligé de faire partie d'une association quelle qu'elle soit.

Article 26. - Tous bénéficient du droit de réunion pacifique et sans armes, que ce soit pour des affaires privées ou pour discuter de questions politiques et examiner la conduite publique des fonctionnaires.

Les réunions dans des locaux privés ne nécessitent pas d'autorisation préalable. Celles qui se déroulent dans des lieux publics seront réglementées par la loi.

80 Article 27. - La liberté de pétition, sous forme individuelle ou collective, à un fonctionnaire ou à un organisme officiel, quels qu'ils soient, et le droit d'obtenir une rapide décision sont garantis.

81 Article 28. - Nul ne peut être inquiété ou poursuivi pour la manifestation de ses opinions, ni pour un acte quelconque qui n'enfreint pas la loi. 
Les actions privées qui ne portent pas atteinte à la morale ou à l'ordre publics, ou qui ne portent pas préjudice à un tiers, échappent à l'action de la loi.

Cependant, les membres du clergé régulier ou séculier ne peuvent se livrer à aucune forme de propagande politique en invoquant des motifs religieux ou en utilisant des croyances religieuses.

Article 29. - Chacun peut communiquer sa pensée par la parole ${ }^{1}$ ou par l'écrit, et par la voie de presse sans subir de censure préalable, mais il sera responsable des actes commis dans l'exercice de ce droit dans les cas et selon les modalités définis par la loi.

Article 30. - Le libre accès aux services administratifs aux fins d'information sur des questions d'intérêt public est garanti.

Sous réserve des secrets de l'Etat.

Article 31. - Le territoire de Costa Rica sera un asile pour toute personne persécutée pour des raisons politiques. Si pour un impératif légal, son expulsion est décrétée, elle ne pourra être renvoyée vers le pays où elle fut persécutée.

L'extradition sera réglementée par la loi ou par les traités internationaux et l'on n'y procèdera jamais en cas de délits politiques ou connexes à ceux-ci, selon la qualification costaricaine.

Article 32. - Aucun Costaricain ne pourra être contraint à quitter le territoire national.

Article 33. - Tous les hommes sont égaux devant la loi et ne pourront être victimes d'une discrimination quelconque contraire à la dignité humaine.

(Réforme constitutionnelle, loi no 7880 du 27 mai 1999)

Article 34. - Aucune loi n'aura d'effet rétroactif au détriment d'une personne, de ses droits patrimoniaux acquis ou de situations juridiques établies.

Article 35. - Nul ne sera jugé par une commission, un tribunal ou juge nommés spécialement pour ce cas, mais doit l'être exclusivement par les tribunaux établis conformément à cette Constitution.

Article 36. - En matière criminelle, nul n'est obligé de témoigner contre lui-même, ni contre son conjoint, ses ascendants, descendants ou parents collatéraux jusqu'au troisième degré compris de parenté de consanguinité ou d'alliance inclus.

Article 37. - Nul ne sera détenu, sans un indice probant d'avoir commis un délit, et sans mandat écrit du juge ou d'une autorité en charge de l'ordre public, excepté quand il s'agit d'un accusé fugitif ou d'un délinquant pris en flagrant délit; dans tous les cas, il devra être mis à la disposition du juge compétent dans un délai de rigueur de 24 heures.

Article 38. - Nul ne pourra être emprisonné pour dette.

7 Article 39. - Nul ne subira de peine, sauf pour délit, quasi-délit ou faute, sanctionnés par une loi antérieure et en vertu d'un jugement sans appel prononcé par l'autorité compétente, à condition que soient reconnus les moyens nécessaires à l'exercice de sa défense et que soit démontrée sa culpabilité.

Be constitue pas une violation à cet article ou aux deux précédents, la contrainte corporelle en matière civile ou en matière de travail ou les détentions qui peuvent être ordonnées en cas d'insolvabilités, faillites ou de liquidations judiciaires. 
99 Article 40. - Nul ne sera soumis à un traitement cruel ou dégradant ni à une peine perpétuelle, ni à une peine de confiscation. Toute déclaration obtenue par la violence sera nulle.

100 Article 41. - Au travers des lois, tous doivent obtenir réparation pour les injures ou dommages qu'ils ont subis dans leur personne, leurs biens ou leurs intérêts moraux. Ils doivent bénéficier d'une justice prompte, de qualité, sans déni de justice et en stricte conformité avec les lois.

101 Article 42. - Un même juge ne peut siéger en diverses instances pour statuer sur un même point. Nul ne pourra être jugé plus d'une fois pour le même fait punissable.

Il est interdit de rouvrir des causes pénales jugées et de remettre en cause des jugements prononcés avec l'autorité de la chose jugée, sauf en cas de recours en révision.

Article 43. - Toute personne a le droit de voir se régler ses différends patrimoniaux par le biais d'arbitres, qu'elle ait ou non déjà intenté un procès.

104 Article 44. - Pour qu'une personne puisse être gardée au secret plus de quarante-huit heures, il est nécessaire d'obtenir une décision judiciaire ; cette procédure pourra être étendue à dix jours consécutifs et en aucun cas n'empêchera l'exercice de l'inspection judiciaire.

Article 45. - La propriété est inviolable; nul ne pourra en être privé, si ce n'est pour un motif d'intérêt public légalement vérifié, accompagné d'une indemnisation préalable conformément à la loi. En cas de guerre ou de troubles intérieurs, il n'est pas indispensable que l'indemnisation soit préalable. Cependant, le prix correspondant sera réglé au plus tard deux années à compter de la fin de l'état d'urgence. Pour des raisons de nécessité publique, l'Assemblée législative, pourra par un vote des deux-tiers de la totalité de ses membres, limiter le droit de propriété dans l'intérêt social.

106 Article 46. - Sont interdits les monopoles privés, et tout acte, même s'il trouve son origine dans une loi, qui menace ou restreint la liberté de commerce, de l'agriculture et de l'industrie.

107 Est d'intérêt public l'action de l'Etat destinée à empêcher toute pratique ou tendance monopolistique.

108 Les entreprises constituées en monopole de fait doivent être soumises à une législation spéciale.

109 Pour établir de nouveaux monopoles en faveur de l'Etat ou des municipalités, il est nécessaire d'avoir l'approbation des $2 / 3$ de la totalité des membres de l'Assemblée législative.

110 Les consommateurs et usagers ont droit à la protection de leur santé, environnement, sécurité et intérêts économiques, à recevoir une information adaptée et véridique ; à la liberté de choix et à un traitement équitable. L'Etat appuiera les organismes que ceux-ci constituent pour la défense de leurs droits. La loi réglemente ces pratiques.

111 (Réforme constitutionnelle, loi $n^{\circ} 7607$ du 29 mai 1996)

112 Article 47. - Tout auteur, inventeur, producteur ou commerçant bénéficiera temporairement de la propriété exclusive sur son œuvre, invention, marque ou nom commercial, conformément à la loi.

113 Article 48. - Toute personne a droit au recours d'habeas corpus pour garantir sa liberté et son intégrité personnelles, et au recours de l'amparo pour maintenir ou rétablir la 
jouissance des autres droits contenus dans cette Constitution, ainsi que ceux de caractère fondamental institués dans les instruments internationaux sur les droits de l'homme, applicables dans la République. Les deux recours seront de la compétence de la Chambre spéciale mentionnée à l'article 10.

(Réforme constitutionnelle, loi $n^{\circ} 7128$ du 18 août 1989)

Article 49. - Est instituée une juridiction du contentieux administratif en tant que fonction du Pouvoir judiciaire avec pour objet de garantir la légalité de la fonction administrative de l'Etat, de ses institutions et de tout autre organisme de droit public.

Le détournement de pouvoir des actes administratifs sera un motif de contestation.

La loi protègera, au moins, les droits personnels et les intérêts légitimes des administrés.

(Réforme constitutionnelle, loi $n^{\circ} 3124$ du 25 juin 1963)

\section{TITRE V}

\section{DROITS ET GARANTIES SOCIALES}

Chapitre unique

Article 50. - L'Etat assurera le plus grand bien-être possible à tous les habitants du pays, en organisant et en stimulant la production et la meilleure répartition de la richesse.

Toute personne a droit à un environnement sain et écologiquement équilibré. Pour cela, il est légitime de dénoncer les actes qui enfreignent ce droit pour réclamer la réparation du dommage causé.

L'Etat garantira, défendra et préservera ces droits. La loi déterminera les responsabilités et les sanctions correspondantes.

(Réforme constitutionnelle, loi $n^{\circ} 7412$ du 3 juin 1994)

Article 51. - La famille, comme élément naturel et comme fondement de la société, a droit à la protection spéciale de l'Etat. Auront également droit à cette protection la mère, l'enfant, le vieillard et le malade sans ressources.

Article 52. - Le mariage est la base fondamentale de la famille et repose sur l'égalité de droits des conjoints.

Article 53. - Les parents sont tenus à l'égard des enfants nés hors du mariage aux mêmes obligations qu'à l'égard de ceux nés dans le cadre du mariage.

Toute personne a le droit de savoir qui sont ses parents, conformément à la loi.

Article 54. - Toute discrimination personnelle effectuée en fonction de la nature de la filiation est interdite.

Article 55. - La protection spéciale de la mère et du mineur sera à la charge d'une institution autonome dénommée Fondation Nationale de l'Enfance ${ }^{2}$, avec la collaboration des autres institutions de l'Etat.

Article 56. - Le travail est un droit de l'individu et une obligation vis-à-vis de la société. L'Etat doit procurer à tous une occupation honnête et utile, correctement rémunérée, et empêcher que à cause de cela ne s'instaurent des conditions qui menacent d'une manière quelconque la liberté ou la dignité de l'homme ou réduisent son travail à la condition de simple marchandise. L'Etat garantit le droit de libre choix du travail. 
Article 57. - Tout travailleur a droit à un salaire minimum, fixé périodiquement, pour une journée de travail normal, qui lui assure le bien-être et une existence digne. Les salaires sont égaux pour des travaux égaux dans des conditions identiques d'efficacité.

Tout ce qui est relatif à la fixation des salaires minimum sera à la charge d'un organisme technique que la loi détermine.

Article 58. - La journée ordinaire de travail diurne ne peut excéder huit heures par jour et quarante-huit heures par semaine. La journée ordinaire de travail nocturne ne peut excéder six heures par jour et trente-six heures par semaine. Le travail en heures supplémentaires doit être rémunéré cinquante pour cent de plus que les gages ou salaires convenus. Cependant, ces dispositions ne s'appliquent pas dans des cas exceptionnels très précis, que détermine la loi.

Article 59. - Tous les travailleurs ont droit à un jour de congé après six jours consécutifs de travail, et à des congés payés annuels dont la durée et la date seront déterminées par la loi, mais qui en aucun cas ne peuvent être inférieurs à deux semaines pour 50 semaines de service continu; le tout sans préjudice des exceptions très précises établies par le législateur.

Article 60. - Tant les chefs d'entreprise que les travailleurs pourront se syndiquer librement, dans le but exclusif d'obtenir et de conserver des avantages économiques, sociaux ou professionnels.

Il est interdit aux étrangers de diriger des syndicats ou d'y exercer des responsabilités.

Article 61. - Le droit de lock-out est reconnu aux chefs d'entreprise comme le droit de grève aux travailleurs, sauf dans les services publics conformément à ce qui est précisé dans la loi pour ce qui les concerne et conformément aux réglementations que celle-ci établira, lesquelles devront interdire tout acte de contrainte ou de violence.

Article 62. - Les conventions collectives de travail qui sont conclues, conformément à la loi, sont négociées entre chefs d'entreprise ou syndicats de chefs d'entreprise et syndicats de travailleurs légalement organisés, auront force loi.

Article 63. - Les travailleurs licenciés sans cause valable ont droit à une indemnité s'il n'existe pas de couverture par une assurance chômage.

Article 64. - L'Etat favorise la création de coopératives, en tant que moyen de favoriser de meilleures conditions de vie aux travailleurs.

Article 65. - L'Etat favorise la construction de logements populaires et crée le patrimoine familial du travailleur.

Article 66. - Tout chef d'entreprise doit adopter dans son entreprise les mesures nécessaires à l'hygiène et à la sécurité du travail.

Article 67. - L'Etat veille à la formation technique et culturelle des travailleurs.

Article 68. - Nul ne pourra introduire de discrimination en matière de salaire, d'avantages ou de conditions de travail entre Costaricains et étrangers, ou en ce qui concerne un groupe quelconque de travailleurs.

A égalité de conditions, les travailleurs costaricains doivent être préférés.

Article 69. - Les contrats de métayage rural seront réglementés de manière à assurer l'exploitation rationnelle de la terre et la répartition équitable de ses produits entre propriétaires et métayers.

8 Article 70. - On établira une juridiction du travail, dépendante du Pouvoir judiciaire. 

dans leur travail.

\section{TITRE VI}

\section{LA RELIGION}

Chapitre unique

loi $n^{\circ} 5703$ du 6 juin 1975)

\section{TITRE VII}

\section{L'EDUCATION ET LA CULTURE}

Chapitre unique

Article 71. - Les lois devront une protection spéciale aux mères et aux enfants mineurs

Article 72. - L'Etat maintiendra, tant qu'il n'existe pas d'assurances-chômage, un système technique permanent de protection des chômeurs involontaires, et veillera à leur réintégration dans le monde du travail.

Article 73. - Sont établies les assurances sociales au bénéfice des travailleurs manuels et intellectuels, organisées par le système de contribution obligatoire de l'Etat, des patrons et travailleurs, afin de protéger ces derniers contre les risques de maladie, d'invalidité, de maternité, de vieillesse, de décès et autres éventualités que la loi détermine.

L'administration et l'organisation des assurances sociales seront à la charge d'une institution autonome, dénommée Caisse Costaricaine de Sécurité Sociale ${ }^{3}$.

Nul ne pourra transférer ni employer à des fins différentes de celles qui ont motivé leur création, les fonds et les réserves des assurances sociales.

Les assurances contre les risques professionnels seront exclusivement à la charge des chefs d'entreprise et seront régies par des dispositions spéciales.

(Réforme constitutionnelle, loi nº 2737 du 12 mai 1961)

Article 74. - Les droits et avantages auxquels ce Chapitre se réfère sont irrévocables. Leur énumération n'est pas exclusive d'autres qui dérivent du principe chrétien de justice sociale et qu'énoncerait la loi ; ils seront applicables sur un principe d'égalité à tous ceux qui participent au processus de production, et réglementés par la législation sociale et la législation du travail, afin de réaliser une politique permanente de solidarité nationale.

Article 75. - La Religion Catholique, Apostolique et Romaine est celle de l'Etat, lequel contribue à son entretien, sans empêcher le libre exercice dans la République des autres cultes, à condition qu'ils ne s'opposent pas à la morale universelle ni aux bonnes mœurs.

(Modification de la numérotation par la réforme constitutionnelle,

Article 76. - L'espagnol est la langue officielle de la Nation. Cependant, l'État veillera à la préservation et au développement des langues indigènes nationales.

(Réforme constitutionnelle, loi n 7878 du 27 mai 1999)

Article 77. - L'instruction publique sera organisée comme un processus intégral en différents cycles, de la pré-scolarité jusqu'à l'université. 
167 Article 78. - L'éducation préscolaire et l'enseignement primaire sont obligatoires. Ceux-ci et l'éducation secondaire dans le système public sont gratuits et pris en charge par la Nation.

Dans l'éducation étatique, incluant le supérieur, la charge publique ne sera pas inférieure à six pour cent $(6 \%)$ par an du Produit Intérieur Brut, conformément à la loi, sans préjudice de ce qui est stipulé aux articles 84 et 85 de la présente Constitution. L'Etat facilitera la poursuite des études supérieures à ceux qui ne disposent pas de ressources financières. L'attribution de bourses et des aides appropriées sera une charge du ministère compétent, par le biais d'un organisme que détermine la loi. (Réforme constitutionnelle $n^{\circ} 7676$ du 23 juillet 1997)

171 Article 79. - La liberté d'enseignement est garantie. Cependant, tout centre d'enseignement privé sera sous le contrôle de l'Etat.

172 Article 80. - L'initiative privée en matière d'éducation méritera d'être encouragée par l'Etat, selon les modalités que déterminera la loi. Article 81. - La direction générale de l'enseignement public appartient à un conseil supérieur constitué conformément à la loi et présidé par le Ministre compétent. 82. - L'Etat procurera de la nourriture et des vêtements aux écoliers indigents, conformément à la loi.

175 Article 83. - L'Etat protège et organise l'éducation des adultes, afin de combattre l'analphabétisme et de proposer une chance de culture à ceux qui désirent améliorer leur condition intellectuelle, sociale et économique.

Article 84. - L'Université de Costa Rica est une institution d'enseignement supérieur qui est indépendante dans l'exercice de ses fonctions et jouit de la pleine capacité juridique pour acquérir des droits et contracter des obligations, ainsi que de choisir sa propre organisation et sa propre direction. Les autres établissements d'enseignement supérieur universitaire de l'Etat bénéficieront de la même indépendance fonctionnelle et d'une égale capacité juridique que l'Université de Costa Rica.

L'Etat les dotera d'un patrimoine propre et participera à leur financement.

(Réforme constitutionnelle, loi $n^{\circ} 5697$ du 9 juin 1975)

Article 85. - L'Etat dotera d'un patrimoine propre l'Université de Costa Rica, l'Institut Technologique de Costa Rica, l'Université Nationale et l'Université d'Etat à Distance et leur créera les revenus propres, indépendamment de ceux qui viennent de ces institutions. De plus, il créera avec les rentes actuelles et avec d'autres qui seraient nécessaires un fonds spécial pour le financement de l'enseignement supérieur d'Etat. La Banque Centrale de Costa Rica administrera ce fonds et, chaque mois, en met un douzième à la disposition des institutions citées, assurera la distribution que détermine le corps en charge de la coordination de l'enseignement supérieur universitaire d'Etat. Les revenus de ce fonds spécial ne pourront être supprimés, ni diminués, si ne sont pas créés, simultanément, d'autres avantages de remplacement.

L'organisme en charge de la coordination de l'enseignement supérieur universitaire de l'Etat préparera un plan national pour cet enseignement, en tenant compte des lignes directrices qu'établira le plan national.

181 Ce plan devra être conclu, au plus tard, le 30 juin des années divisibles par cinq et couvrir le quinquennat immédiat suivant. Il devra inclure tant les dépenses de roulement que les 
dépenses d'investissement qui seront considérées nécessaires pour le bon fonctionnement des institutions mentionnées au présent article.

Le Pouvoir exécutif inclura au budget ordinaire des dépenses de la République, la partie correspondante, mentionnée dans le plan, adoptée conformément à la variation du pouvoir d'achat de la monnaie.

Tout différend qui surgira, en rapport avec l'approbation du montant budgétaire du Plan national d'Enseignement Supérieur de l'Etat, sera réglé par l'Assemblée législative.

(Réforme constitutionnelle, loi $n^{\circ} 6580$ du 18 mai 1981)

Article 86. - L'Etat formera un corps d'enseignants professionnels par le biais d'institutions spéciales à l'Université du Costa Rica et dans les autres institutions d'enseignement supérieur universitaire.

(Réforme constitutionnelle, loi $n^{\circ} 5697$ du 9 juin 1975)

Article 87. - La liberté de la chaire ${ }^{4}$ est un principe fondamental de l'enseignement universitaire.

Article 88. - Lors de la discussion et de l'adoption de projets de loi relatifs à des questions relevant de la compétence de l'Université du Costa Rica et des autres institutions d'enseignement supérieur universitaire, ou qui s'y rapportent directement, l'Assemblée législative devra entendre préalablement le Conseil de l'université ou l'organe de direction correspondant de chacun de ces établissements.

(Réforme constitutionnelle, loi $n^{\circ} 5697$ du 9 juin 1975)

Article 89. - Parmi les objectifs culturels de la République il y a la protection des beautés naturelles, la conservation et le développement du patrimoine historique et artistique de la Nation et l'encouragement à l'initiative privée en vue du progrès scientifique et artistique.

\section{TITRE VIII}

\section{DROITS ET DEVOIRS POLITIQUES}

Chapitre I

Les Citoyens

Article 90. - La citoyenneté est la conjonction de droits et de devoirs politiques concernant des Costaricains âgés de plus dix-huit ans.

(Réforme constitutionnelle, loi nº 4763 du 17 mai 1971)

Article 91. - La citoyenneté est seulement suspendue :

1) Par interdiction judiciairement déclarée ;

2) Par un jugement infligeant une peine de suspension des droits politiques.

Article 92.- La citoyenneté se recouvre dans tous les cas et selon les procédures déterminées par la loi.

Chapitre II

Le droit de vote 
 politiques aux processus électoraux, et satisfaire aux nécessités de capacité et d'organisation politique. Chaque parti politique fixera les pourcentages correspondants à ces rubriques.

219 2) Auront droit à la contribution de l'Etat, les partis politiques qui participeront au processus électoral en application de cet article et obtiendront au moins quatre pour cent (4\%) des suffrages valablement exprimés à un scrutin national ou des inscrits à un scrutin provincial, qui obtiennent au minimum ce pourcentage en la province ou l'élection, pour le moins, d'un député.

3) Avant l'octroi des garanties correspondantes, les partis politiques auront droit à ce que soit avancée une partie de la contribution étatique, selon ce que déterminera la loi.

4) Pour recevoir la part de l'Etat, les partis devront présenter leurs comptes au Tribunal Suprême Electoral.

Pouvoirs dans la Caraïbe, 16 | 2010 
Les contributions privées aux partis politiques seront soumises au principe de publicité et seront réglementées par la loi.

La loi qui établira les procédures, les moyens de contrôle et de plus la réglementation pour l'application du présent article, nécessitera, pour son adoption et sa réforme, le vote des deux-tiers du total des membres de l'Assemblée législative.

(Réforme constitutionnelle, loi $n^{\circ} 7675$ du 2 juillet 1997)

Article 97. - Lors de la discussion et de l'approbation des projets de loi relatifs aux matières électorales, l'Assemblée législative devra consulter le Tribunal Suprême Electoral ; pour écarter son avis, il sera nécessaire qu'il y ait un vote des deux-tiers de ses membres.

Au cours des six mois qui précèdent et des quatre mois postérieurs à la tenue d'une élection populaire, l'Assemblée législative ne pourra, cependant, adopter des projets de loi sur ces questions en qui auraient été désapprouvés par le Tribunal Suprême Electoral.

Article 98. - Les citoyens auront le droit de se grouper en partis pour intervenir dans la politique nationale, pourvu que les partis s'engagent dans leurs programmes à respecter l'ordre constitutionnel de la République. Les partis politiques expriment le pluralisme politique, concourent à la formation et la manifestation de la volonté populaire et sont des instruments fondamentaux pour la participation politique. Leur création et l'exercice de leur activité seront libres dans le cadre du respect de la Constitution et de la loi. Leur structure interne et leur fonctionnement seront démocratiques.

(Réforme constitutionnelle, loi $n^{\circ} 7675$ du 2 juillet 1997)

Chapitre III

Le Tribunal Suprême Electoral

Article 99. - L'organisation, la direction et la surveillance des opérations électorales, sont de la compétence exclusive du Tribunal Suprême Electoral, lequel jouit de l'indépendance totale dans l'accomplissement de sa mission. Du Tribunal dépendent les autres organismes électoraux.

Article 100. - Le Tribunal Suprême Electoral sera constitué, ordinairement de trois magistrats titulaires et six suppléants nommés par la Cour suprême de Justice à la majorité des deux-tiers de ses membres. Ils devront satisfaire aux mêmes conditions et seront soumis aux mêmes responsabilités que les magistrats qui constituent la Cour.

Pendant l'année qui précède et les six mois après la tenue des élections générales pour la Présidence de la République ou des Députés à l'Assemblée législative, le Tribunal Suprême Electoral devra augmenter de deux le nombre de ses magistrats suppléants pour former, pendant cette période, un tribunal de cinq membres.

34 Les magistrats du Tribunal Suprême Electoral seront soumis aux conditions de travail, en ce qu'elles leur sont applicables, et au temps minimum de travail par jour qu'indiquera la Loi organique sur le pouvoir judiciaire pour les magistrats de la Cour de cassation, et percevront les mêmes rémunérations qui seront fixées par celle-ci.

(Réforme constitutionnelle, loi n 3513 du 24 juin 1965)

Article 101. - Les magistrats du Tribunal Suprême Electoral resteront en fonction six années. Un titulaire et deux suppléants devront être renouvelés chaque deux années, mais ils sont rééligibles. 
Les magistrats du Tribunal Suprême Electoral bénéficieront des mêmes immunités et prérogatives que les membres des pouvoirs suprêmes.

(Réforme constitutionnelle, loi $n^{\circ} 3513$ du 24 juin 1965)

Article 102. - Le Tribunal Suprême Electoral assure les fonctions suivantes :

1) Convoquer les élections populaires ;

2) Nommer les membres des bureaux électoraux ${ }^{6}$, conformément à la loi :

3) Interpréter de façon exclusive et obligatoire les dispositions constitutionnelles et législatives afférentes à la matière électorale ;

4) Connaître des recours en appel des décisions prises par l'Etat civil et les Juntes Electorales;

5) Enquêter par lui-même ou par le biais de délégués, et se prononcer sur les réclamations formulées par les partis en ce qui concerne la partialité politique des agents de l'Etat dans l'exercice de leurs fonctions, ou sur les activités politiques des fonctionnaires à qui il est interdit d'en exercer. Tout constat de culpabilité prononcé par le Tribunal entraînera obligatoirement la destitution et met le coupable dans l'incapacité d'exercer des fonctions publiques pour une période qui ne peut être inférieure à deux années, sans préjudice des responsabilités pénales qui pourront être encourues. Nonobstant, si l'enquête menée relève des charges contre le Président de la République, les ministres, les représentants diplomatiques, le contrôleur et le Sous-contrôleur généraux de la République, ou les magistrats de la Cour suprême de Justice, le Tribunal se limitera à communiquer à l'Assemblée législative le résultat de l'enquête ;

6) Edicter, en ce qui concerne la force publique, les mesures nécessaires pour que le processus électoral se déroule sans aucune restriction de garanties et de liberté. Au cas où ce qui est décrété touche le recrutement militaire, le Tribunal pourra également édicter, les mesures adéquates pour que ne soit pas perturbé le processus électoral, afin que tous les citoyens puissent émettre librement leur vote. Ces mesures peuvent être prises par le tribunal lui-même ou par des délégués qu'il désigne ;

7) Faire le compte définitif des suffrages exprimés pour l'élection du Président et du viceprésident de la République, des députés à l'Assemblée législative, des membres des municipalités et des représentants aux Assemblées constituantes ;

8) Assurer la proclamation définitive de l'élection du Président et du vice-président de la République, dans les trente jours suivant la date de l'élection, et des autres fonctionnaires cités à l'alinéa précédent, dans le délai prévu par la loi ;

9) Organiser, diriger, contrôler, scruter et déclarer les résultats des processus référendaires. Il ne pourra pas être convoqué plus d'un referendum par an ; ni pendant les six mois précédents ou postérieurs à l'élection présidentielle. Les résultats s'imposent à l'État si participe, au moins, trente pour cent $(30 \%)$ des citoyens inscrits sur les listes électorales, pour la législation ordinaire, et quarante pour cent (40\%) au moins, pour les réformes partielles de la Constitution et les affaires qui requièrent une approbation législative par majorité qualifiée.

(Réforme constitutionnelle, loi nº 8281 du 28 mai 2002)

10) Les autres attributions que lui confèrent cette Constitution et les lois.

Article 103. - Les décisions du Tribunal Suprême Electoral sont sans recours, sauf en cas de forfaiture. 
Article 104. - L'état civil dépend exclusivement du Tribunal Suprême Electoral. Ses attributions sont les suivantes :

1) Tenir le Registre Central de l'Etat civil et constituer la liste des électeurs ;

2) Statuer sur les demandes pour acquérir ou récupérer la qualité de Costaricain, ainsi que sur les cas de perte de nationalité ; exécuter les sentences judiciaires qui suspendent la citoyenneté et statuer sur les démarches pour la recouvrer. Les décisions qu'adopte l'état civil conformément aux compétences définies par la présente sous-section sont susceptibles d'appel devant le Tribunal Suprême Electoral ;

3) Délivrer les cartes d'identité ;

4) Les autres attributions prévues par la présente Constitution et par les lois.

\section{TITRE IX}

\section{LE POUVOIR LEGISLATIF}

Chapitre 1

L'Organisation de l'Assemblée législative

Article 105. - Le pouvoir de légiférer appartient au peuple, lequel le délègue, par le biais du suffrage, à l'Assemblée législative. Un tel pouvoir ne pourra être subordonné ni soumis à des limitations, moyennant une quelconque convention ou contrat, ni directement ni indirectement, sauf en vertu de traités, conformément aux principes du droit international.

Le peuple pourra également exercer ce pouvoir par voie de referendum, pour approuver ou abolir des lois et des réformes partielles de la Constitution, à l'initiative d'au moins cinq pour cent $(5 \%)$ des citoyens inscrits sur les listes électorales; l'Assemblée Législative, par l'approbation des deux tiers de ses membres, ou le Pouvoir Exécutif avec la majorité absolue de la totalité des membres de l'Assemblée Législative.

Le referendum ne conviendra pas si les projets sont relatifs au domaine budgétaire, fiscal, monétaire ou aux contributions, au crédit, aux pensions, à la sécurité, à l'approbation de prêts et contrats ou actes de nature administrative.

Cet institut sera réglé par loi, approuvée par les deux tiers de la totalité des membres de l'Assemblée Législative.

(Réforme constitutionnelle, loi $n^{\circ} 8281$ du 28 mai 2002)

Article 106. - Les députés tiennent leur fonction de la Nation et sont élus par province.

L'Assemblée se compose de cinquante-sept députés. Chaque fois qu'est réalisé un recensement général de la population, le Tribunal Suprême Electoral fixe le nombre de députés par province, proportionnellement à la population de chacune d'entre elles.

(Réforme constitutionnelle, loi $n^{\circ} 2741$ du 12 mai 1961)

Article 107. - Les députés ont un mandat de quatre années et ne seront pas immédiatement rééligibles.

Article 108. - Pour être député, il faut :

1) Jouir de ses droits de citoyen ;

2) Etre costaricain par naissance, ou par naturalisation avec dix années de résidence dans le pays depuis l'obtention de la nationalité ; 


\section{3) Avoir atteint l'âge de vingt-et-un ans.}

Article 109. - Ne peuvent être élus députés, ni inscrits comme candidats à cette fonction :

1) Le Président de la République ou toute personne l'ayant remplacé dans l'exercice de la Présidence au moment de l'élection ;

2) Les ministres ;

3) Les magistrats titulaires de la Cour suprême de Justice ;

4) Les magistrats titulaires et suppléants du Tribunal Suprême Electoral, et le Directeur du bureau d'état civil ;

5) Les militaires en service actif ;

6) Ceux qui exercent leur juridiction, un pouvoir civil ou de police, sur une province ;

7) Les directeurs des institutions autonomes;

8) Les parents de celui qui occupe la présidence de la République jusqu'au second degré de consanguinité ou alliance, inclus.

Ces incompatibilités affecteront ceux qui remplissent les charges indiquées au cours des six mois précédents l'élection.

Article 110. - Le député n'est pas responsable des opinions qu'il émet à l'Assemblée. Durant les sessions, il ne pourra être arrêté pour cause civile, sauf autorisation de l'Assemblée ou si le député y consent.

A partir du moment où il a été déclaré élu titulaire ou suppléant, jusqu'à ce qu'il ait achevé son mandat, il ne pourra être privé de sa liberté pour un motif pénal, sauf quand sa suspension a été préalablement décidée par l'Assemblée. Cette immunité ne jouera pas en cas de flagrant délit, ou lorsque le député y renonce. Cependant, le député qui aura été détenu en cas de flagrant délit, sera remis en liberté si l'Assemblée l'ordonne.

Article 111. - Nul député ne pourra accepter, après avoir prêté serment, sous peine de perdre son mandat, de charges ou emplois des autres Pouvoirs de l'Etat ou des institutions autonomes, sauf quand il s'agit d'un ministère. Dans ce cas, il est réintégré à l'Assemblée à la cessation de ses fonctions.

Cette interdiction ne concerne pas ceux qui seraient appelés à faire partie de délégations internationales, ou exerçant des charges dans les institutions de bienfaisance, ou qui seraient titulaires de chaires de l'Université de Costa Rica ou dans d'autres institutions d'enseignement supérieur de l'Etat.

\section{(Réforme constitutionnelle, loi no 5697 du 9 juin 1975)}

Article 112. - La fonction législative est aussi incompatible avec l'exercice de toute autre charge publique élective.

9 Les députés ne pourront conclure, ni directement ni indirectement, ou par représentation, un contrat quelconque avec l'Etat, ni obtenir de concession sur le domaine public impliquant privilège, ni intervenir en tant que directeurs, administrateurs ou gérants d'entreprises qui contractent avec l'Etat, pour des travaux, fournitures ou l'exploitation de services publics.

La violation de l'une des interdictions mentionnées au présent article ou l'article précédent, conduira à la perte du mandat de député. Il en sera de même si, dans l'exercice d'un ministère, le député viole l'une quelconque de ces interdictions. 
Article 113. - La loi fixera la rémunération et les aides techniques et administratives qui sont accordées aux députés.

(Réforme constitutionnelle, loi $n^{\circ} 6960$ du $1^{\text {er }}$ juin 1984)

Article 114. - L'Assemblée résidera dans la capitale de la République. Un vote des deuxtiers de ses membres sera requis pour déplacer son siège en un autre lieu ou pour suspendre ses sessions pour un temps déterminé.

Article 115. - L'Assemblée élira son Bureau au début de chaque législature.

Le Président et le vice-président doivent réunir les mêmes conditions d'éligibilité exigées que pour être Président de la République. Le président de l'Assemblée prêtera serment devant celle-ci et les députés devant le président.

Article 116. - L'Assemblée législative se réunira chaque année le premier jour de mai, même quand elle n'a pas été convoquée, et ses sessions ordinaires dureront six mois, divisées en deux périodes:

Du premier mai au trente et un juillet et du premier septembre au trente novembre.

Une législature comprend les sessions ordinaires et extraordinaires tenues entre le $1^{\mathrm{er}}$ mai et le 30 avril suivant.

Article 117. - L'Assemblée ne pourra tenir ses séances sans la présence des deux-tiers de ses membres.

Si, le jour prévu, il était impossible de commencer les séances, ou si l'ouverture ne pouvait se poursuivre faute de quorum, les membres présents adresseront une injonction aux absents, sous peine de sanctions prévues par le Règlement, de participer, et l'Assemblée ouvrira ou poursuivra ses séances lorsque le quorum sera atteint.

Les séances seront publiques sauf si, pour des motifs très précis et d'intérêt général, il est décidé de se réunir à huis clos, et ce à la majorité des deux-tiers au moins des députés présents.

Article 118. - Le pouvoir exécutif pourra convoquer l'Assemblée législative en sessions extraordinaires. Dans ce cas, ne seront pas examinées des matières différentes de celles mentionnées dans le décret de convocation, excepté s'il s'agit de la nomination de fonctionnaires relevant de l'Assemblée ou de réformes législatives qui s'avèrent indispensables pour résoudre des questions soumises à son examen.

Article 119. - Les décisions de l'Assemblée se prendront à la majorité absolue des votants présents, excepté les cas où cette Constitution exige une majorité plus importante.

Article 120. - Le pouvoir exécutif mettra à la disposition de l'Assemblée législative, les forces de police que le président de celle-ci sollicite.

Chapitre II

Attributions de l'Assemblée législative

Article 121. - En plus des autres attributions que lui confère cette Constitution, il appartient exclusivement à l'Assemblée législative :

1) D’adopter les lois, les réformer, y déroger et en donner une interprétation authentique, sauf celles énoncées au chapitre relatif au Tribunal Suprême Electoral ;

2) De désigner le lieu de ses sessions, les ouvrir et les clore, les suspendre et les poursuivre comme il conviendra ;

3) De nommer les magistrats titulaires et suppléants de la Cour suprême de Justice ; 
311 4) D'approuver ou rejeter les conventions internationales, les traités publics et les concordats.

Les traités publics et conventions internationales, qui attribuent ou transfèrent des compétences déterminées à une organisation juridique communautaire, avec la proposition de réaliser des objectifs régionaux et communs, requièrent l'approbation de l'Assemblée législative, à la majorité des deux-tiers au moins de la totalité de ses membres.

313 Ne requièrent pas l'approbation législative les protocoles de moindre importance, dérivés des traités publics ou des conventions internationales approuvés par l'Assemblée, quand ces instruments autorisent de façon exprès une telle procédure dérivée.

(Réforme constitutionnelle, loi $n^{\circ} 4123$ du 31 mai 1968)

5) De donner ou non son assentiment pour l'entrée de troupes étrangères sur le territoire national et au stationnement de vaisseaux de guerre dans les ports et aérodromes ;

6) D'autoriser le pouvoir exécutif à déclarer l'état de défense nationale et à conclure la paix ;

7) De suspendre à la majorité des deux-tiers au moins de la totalité de ses membres, en cas de nécessité publique évidente, les droits et garanties individuelles mentionnées aux articles 22, 23, 24, 26, 28, 29, 30 et 37 de cette Constitution. Cette suspension pourra être de tout ou partie de ces droits et garanties, pour la totalité ou sur une partie du territoire, et ce pour trente jours au plus; pendant cette période et à l'égard des personnes, le Pouvoir exécutif pourra seul ordonner leur détention dans des établissements non destinés à des détenus de droit commun ou les astreindre à résider dans des lieux habités.

Il devra également rendre compte à l'Assemblée à sa prochaine réunion des mesures prises pour sauvegarder l'ordre public ou maintenir la sécurité de l'Etat. En aucun cas, ne peuvent être suspendus les droits et garanties individuels non mentionnés dans cet alinéa.

319 8) De recevoir le serment légal et d'enregistrer les démissions des membres des Pouvoirs Suprêmes, à l'exception des ministres; de résoudre les problèmes qui surviennent en cas d'incapacité physique ou mentale de celui qui exerce la présidence de la République, et de décider s'il doit confier l'exercice du pouvoir à celui qui devra légalement lui être substitué ;

320 9) Admettre ou non les accusations portées contre celui qui exerce la présidence de la République, les vice-présidents, les membres des Pouvoirs Suprêmes et les représentants diplomatiques, en décidant à la majorité des deux-tiers des membres composant l'Assemblée s'il y a ou non lieu d'engager une action contre eux, les mettant, en cas de réponse affirmative, à la disposition de la Cour suprême de Justice pour jugement ;

321 10) D'ordonner la suspension des fonctionnaires mentionnés à l'alinéa précédent quand il y a lieu d'engager des procédures contre eux pour délit de droit commun ;

322 11) De fixer les budgets ordinaires et extraordinaires de la République ;

323 12) de nommer le Contrôleur et Sous-Contrôleur Généraux de la République ;

13) D'établir les impôts et contributions de niveau national, et autoriser ceux de niveau municipal ;

14) De décider l'aliénation ou l'affectation à l'usage public des biens propres de la Nation.

Ne pourront sortir définitivement du domaine de l'Etat : 
a) L'énergie qui pourra être obtenue des eaux du domaine public sur le territoire national ;

b) Les gisements de charbon, les puits et gisements de pétrole, et autres substances provenant des hydrocarbures, ainsi que les dépôts de minerais radioactifs existant sur le territoire national ;

c) Les services de télécommunications;

Les biens mentionnés aux sous-sections a), b) et c) précédentes ne pourront être exploités que par l'administration publique ou par des particuliers, conformément à la loi ou en vertu d'une concession spéciale accordée pour une durée limitée et conformément aux conditions et clauses établies par l'Assemblée législative.

Les chemins de fer, les installations portuaires et les aéroports nationaux - ces derniers quand ils sont utilisés- ne pourront être aliénés, loués ni grevés de charges, directement ou indirectement, ni échapper sous une forme quelconque au domaine et au contrôle de l'Etat.

15) D'approuver ou rejeter les emprunts ou contrats similaires qui concernent les finances publiques, conclus par le pouvoir exécutif.

Pour contracter des emprunts à l'étranger ou des emprunts qui, bien que conclus dans le pays, sont financés par des capitaux étrangers, il est précisé que le projet correspondant devra être approuvé à la majorité des deux-tiers des voix des membres de l'Assemblée législative.

(Réforme constitutionnelle, loi no 4123 du 31 mai 1968)

D'attribuer la citoyenneté honorifique pour services exceptionnels rendus à la République, et décréter les honneurs à la mémoire des personnes dont les actions éminentes et hauts faits accréditent cette distinction ;

17) De fixer le contenu de l'unité monétaire et légiférer sur la monnaie, le crédit, les poids et mesures. Pour déterminer le contenu de l'unité monétaire, l'Assemblée devra recueillir préalablement l'avis de l'organe technique chargé de la réglementation monétaire ;

18) De promouvoir le progrès des sciences et des arts et d'assurer pour une période limitée, à tous les auteurs et inventeurs, la propriété de leurs œuvres et inventions respectives ;

19) De créer des établissements pour l'enseignement et le progrès des sciences et des arts, en leur accordant des subventions de soutien et de s'efforcer, en particulier, de généraliser l'enseignement primaire ;

20) Créer les tribunaux et autres organismes pour le service national ;

21) D'accorder à la majorité des deux-tiers au moins de ses membres, l'amnistie et la grâce pour les délits politiques, à l'exception des délits électoraux, qui ne peuvent faire l'objet d'aucune mesure de grâce ;

22) D'adopter un règlement pour son fonctionnement interne, lequel, après avoir été adopté, ne pourra être modifié qu'à la majorité des deux-tiers au moins de ses membres ; 23) De nommer en son sein des Commissions pour enquêter sur toute affaire que l'Assemblée leur confie et remettre un rapport sur ce sujet ;

Les commissions auront libre accès à tous les services officiels pour réaliser leurs enquêtes et se procurer les renseignements qu'elles jugeront nécessaires. Elles pourront 
recevoir les preuves de toute nature et faire comparaitre toute personne devant elles pour l'interroger ;

24) D'interpeller les ministres et, de plus, par un vote des deux-tiers des membres présents, censurer ceux-ci, quand, de l'avis de l'Assemblée, ils sont coupables d'actes inconstitutionnels ou illégaux, ou d'erreurs graves qui ont causé ou peuvent causer un préjudice évident aux intérêts publics.

Font exception dans les deux cas les questions qui font l'objet de négociations diplomatiques ou qui concernent des opérations militaires en cours.

Article 122. - Il est interdit à l'Assemblée de voter par applaudissements les actes officiels, ainsi que d'imposer à la charge du Trésor Public des obligations qui n'ont pas été préalablement déclarées telles par le pouvoir judiciaire ou acceptés par le pouvoir exécutif, ou d'octroyer des bourses, des pensions, des retraites ou des gratifications.

Chapitre III

Article 123. - Pendant les sessions ordinaires, l'initiative des lois appartient à chacun des membres de l'Assemblée législative et au pouvoir exécutif, par le biais des ministres du Gouvernement et à cinq pour cent $(5 \%)$ au moins, des citoyens inscrits sur les listes électorales, si le projet est d'initiative populaire.

L'initiative populaire ne conviendra pas s'il s'agit de projets relatifs au domaine budgétaire, fiscal, monétaire ou aux contributions, au crédit, aux pensions, à l'approbation de prêts et contrats ou actes de nature administrative

projets de loi d'initiative populaire devront être définitivement votés dans le délai impératif indiqué par la loi, à l'exception des projets de loi de réforme constitutionnelle qui doivent se conformer à la procédure prévue à l'article 195 de la Constitution.

Une loi adoptée par les deux tiers des membres de l'Assemblée Législative, déterminera la procédure, les formalités et les autres conditions que doivent remplir les projets de loi d'initiative populaire.

(Réforme constitutionnelle, loi nº 8281 de 28 mai 2002)

Article 124. - Tout projet pour devenir loi doit faire l'objet de deux débats, chacun se déroulant un jour différent et ne se suivant pas, obtenir l'approbation de l'Assemblée et la sanction du pouvoir exécutif; en outre, il doit être publié au Journal officiel, sans préjudice des formalités que cette Constitution requiert tant pour des cas spéciaux que pour ceux qui résultent de l'initiative populaire ou d'une procédure référendaire, selon les articles 102, 105,123, 129 et 195 de cette Constitution. N'ont pas le caractère de loi, et, de ce fait, ne requièrent pas les démarches précédentes, les mesures prises dans l'exercice des attributions énumérées aux alinéas 2), 3), 5), 6), 7), 8), 9), 10), 12), 16), 21), 22), 23) et 24) de l'article 121 ainsi que l'acte législatif organisant un referendum, lesquels sont adoptés en une seule séance et devront être publiés au Journal officiel.

(Réforme constitutionnelle, loi $n^{0} 8281$ de 28 mai 2002)

L'Assemblée législative pourra déléguer, aux commissions permanentes, la connaissance de l'approbation des projets de loi. Cependant, l'Assemblée pourra évoquer, à tout moment, débattre et voter des projets qui avaient fait l'objet de délégation.

Il ne peut être procédé à la délégation en cas d'examen de projets de loi relatifs à la matière électorale, à la création d'impôts nationaux ou à la modification de ceux 
existants, à l'exercice des facultés prévues aux alinéas 4), 11), 14), 15) et 17) de l'article 121 de la Constitution, à la convocation d'une Assemblée constituante, pour un quelconque objet et à la révision partielle de la Constitution.

L'Assemblée nommera les commissions permanentes avec un plein pouvoir législatif, de manière que leur composition reflète, proportionnellement, le nombre de députés des partis politiques qui la composent. La délégation devra être approuvée par la majorité des deux-tiers des membres de l'assemblée, et l'évocation, par la majorité absolue des députés présents.

Le Règlement de l'Assemblée définit le nombre de ces commissions et les conditions pour la délégation et l'évocation, ainsi que les procédures qui s'appliqueront dans ces cas.

L'approbation législative de contrats, accords et autres actes de nature administrative, ne confère pas à ces actes de caractère législatif, même si cette approbation est obtenue selon la procédure ordinairement appliquée à celle-ci.

(Réforme constitutionnelle, loi $n^{\circ} 7347 \mathrm{du} 1^{\text {er }}$ juillet 1993)

Article 125. - Si le pouvoir exécutif n'approuve pas le projet de loi voté par l'Assemblée, il peut opposer son veto et le renvoyer accompagnés des objections pertinentes. Il ne peut être procédé au veto lorsque le projet qui est approuvé est le budget ordinaire de la République.

Article 126. - Dans les dix jours ouvrables comptés à partir de la date de réception d'un projet de loi approuvé par l'Assemblée législative, le pouvoir exécutif pourra formuler des objections s'il le juge inadéquat ou croit nécessaire de l'amender; dans ce dernier cas, il devra proposer les amendements en le renvoyant. Sans objection de sa part pendant ce délai, le Pouvoir exécutif ne pourra s'abstenir de l'approuver et de le publier.

Article 127. - Lorsqu'un projet de loi est réexaminé par l'Assemblée, avec les observations du Pouvoir exécutif, si l'Assemblée repousse celles-ci et si le projet est à nouveau voté à la majorité des deux-tiers de ses membres, il sera réputé adopté de ce fait et il devra être exécuté comme loi de la République. Si les modifications proposées sont adoptées, le projet sera transmis au Pouvoir exécutif qui ne pourra refuser de l'approuver. Si elles sont repoussées ou si le projet ne réunit pas la majorité des deux-tiers des voix, il sera retiré et ne pourra être représenté jusqu'à la législature suivante.

Article 128. - Si l'objection se fonde sur des raisons d'inconstitutionnalité écartées par l'Assemblée législative, le décret législatif sera adressé à la Chambre indiquée à l'article 10 , pour que le différend soit résolu dans les trente jours calendaires suivant la date à laquelle a été reçu l'envoi. Si la Chambre déclare que le projet comporte des dispositions inconstitutionnelles, ces dispositions seront considérées comme rejetées et les autres dispositions seront transmises à l'Assemblée législative pour la poursuite de la procédure. Il en sera de même de l'ensemble du projet de loi approuvé par l'Assemblée législative, si la Chambre déclare qu'il ne contient pas de dispositions inconstitutionnelles.

(Réforme constitutionnelle, loi n⿳0 7128 du 18 août 1989)

Article 129. - Les lois sont obligatoires et prennent effet à compter du jour qu'elles précisent ; à défaut de cette précision, dix jours à compter de leur publication au Journal officiel.

Nul ne peut alléguer son ignorance de la loi sauf dans les cas autorisés par celle-ci.

Toute renonciation aux lois en général ou à celles qui sont d'intérêt public en particulier est sans effet. 

l'observer.

\section{TITRE X}

\section{LE POUVOIR EXECUTIF}

\section{Chapitre 1}

2) Etre d'état laïc ; constitutionnel. cette période ; République. ces fonctions.

Les actes et conventions contraires aux lois prohibitives seront nuls et non avenus, sauf dispositions contraires prévues par la loi.

La loi ne pourra être abrogée ni modifiée, sauf par une autre loi postérieure; et il n'est pas possible d'invoquer la désuétude, la coutume ou une pratique contraire pour ne pas

Par le biais de referendum, le peuple pourra l'abroger ou l'abolir, conformément à l'article 105 de cette Constitution.

(Réforme constitutionnelle, loi $n^{e} 8281$ du 28 mai 2002)

Le Président et les vice-présidents de la République

Article 130. - Le Pouvoir exécutif est exercé, au nom du peuple, par le Président de la République et les ministres en qualité de collaborateurs subordonnés.

Article 131. - Pour être Président ou vice-président de la République, il faut :

1) Etre costaricain par la naissance et citoyen en exercice ;

3) Etre âgé d'au moins trente années.

Article 132. - Ne pourront être élus Président ni vice-président :

1) Le Président ayant exercé la présidence pour quelque durée, le vice-président ou toute personne le remplaçant qui l'aurait assurée pendant la majeure partie d'un mandat

(Réforme constitutionnelle, loi $n^{\circ} 4349$ du 11 juillet 1969)

2) Le vice-président qui aurait conservé cette qualité dans les douze mois précédant l'élection, et qui aurait, en tant que tel, assuré la présidence à un moment quelconque de

3) Tout ascendant ou descendant par consanguinité ou alliance, tout frère de la personne qui occupe la présidence de la République au moment de l'élection ou de celle qui l'aurait occupée à un moment quelconque au cours des douze mois antérieurs à cette élection ;

4) Celui qui aura été ministre durant les douze mois précédant l'élection ;

5) Les magistrats titulaires de la Cour suprême de Justice, les magistrats titulaires et suppléants du Tribunal Suprême Electoral, le directeur de l'état civil, les directeurs ou gérants des institutions autonomes, le Contrôleur et le Sous-Contrôleur Généraux de la

Cette incompatibilité s'appliquera aux personnes qui ont rempli les charges indiquées dans les douze mois précédant la date de l'élection.

Article 133. - L'élection du Président et des vice-présidents se déroulera le premier dimanche de février de l'année au cours de laquelle doit avoir lieu le renouvellement de

Article 134. - Le mandat présidentiel a une durée de quatre années. 

tels actes sera imprescriptible. de justice. suffrages valablement exprimés. élire. suffrages. respectifs de sa liste.

\section{Chapitre II}

Les actes des fonctionnaires et des particuliers qui violent le principe de l'alternance à la Présidence, ou celui de la libre succession présidentielle, consacrés par cette Constitution, sont considérés comme une trahison envers la République. La responsabilité résultant de

Article 135. - Il existe deux vice-présidents de la République, lesquels remplacent le Président, en cas d'absence illimitée de celui-ci, dans l'ordre de leur nomination. De plus, en cas d'absence temporaire, le Président pourra appeler l'un quelconque des viceprésidents pour le remplacer. Quand aucun des vice-présidents ne peut remplir les absences temporaires ou définitives du Président, c'est le Président de l'Assemblée législative qui exercera cette charge.

Article 136. - Le Président et les vice-présidents de la République prendront possession de leur charge le $8^{\text {ème }}$ jour du mois de mai ; et ils quitteront leur fonction dans les mêmes conditions au terme du délai constitutionnel.

Article 137. - Le Président et les vice-présidents prêteront serment devant l'Assemblée législative ; mais s'ils ne peuvent le faire devant elle, ils le feront devant la Cour suprême

Article 138. - Le Président et les vice-présidents seront élus simultanément à la majorité des suffrages, celle-ci devant excéder les quarante pour cents du nombre total de

Les candidats d'un parti à la présidence et aux vice-présidences, doivent figurer pour cette élection sur une même liste nominative, à l'exclusion de toute autre personne à

Si aucune des listes n'atteint la majorité requise, il sera procédé à une seconde élection le premier dimanche d'avril de la même année entre les deux listes qui ont obtenu le plus de voix, et seront élus ceux qui figurent sur la liste ayant obtenu le plus grand nombre de

9 Si au cours de ces élections, deux listes arrivent à égalité de suffrages suffisants, sera considéré élu Président le candidat le plus âgé et comme vice-présidents les candidats

Les citoyens figurant sur une liste déjà inscrite conformément à la loi, ne peuvent renoncer à leur candidature pour la présidence ou la vice-présidence les candidats des deux listes qui ont obtenu le plus grand nombre de voix à la première ne peuvent pas non plus s'abstenir de participer à la seconde élection.

Devoirs et attributions de ceux qui exercent le pouvoir exécutif

Article 139. - Celui qui exerce les fonctions de Président de la République a les attributions et les devoirs exclusifs suivants :

1) Nommer et révoquer librement les ministres;

2) Représenter la Nation dans les actes de caractère officiel ;

3) Exercer le commandement suprême de la force publique ;

4) Présenter à l'Assemblée législative, au début de la première session de l'année, un message écrit relatif aux diverses affaires de l'Administration et à l'état politique de la République, dans lequel, devront être proposées, par surcroît, les mesures qu'il juge 
nécessaires pour la bonne marche du gouvernement, le progrès et le bien-être de la Nation ;

5) Informer préalablement l'Assemblée législative, quand il se propose de sortir du pays, des motifs de son voyage.

(Réforme constitutionnelle, loi $n^{\circ} 7674$ du 17 juin 1997)

Article 140. - Le Président et le ministre du gouvernement concerné assument conjointement les pouvoirs et devoirs suivants :

411 1) Nommer et révoquer librement les membres de la force publique, les employés et les fonctionnaires qui occupent des postes de confiance, et ceux que détermine, dans des cas très précis, la Loi sur la fonction publique ;

2) Nommer et révoquer, dans les conditions prévues par la Loi sur le service civil, les autres agents qui dépendent d'eux;

3) Sanctionner et promulguer les lois, prendre les règlements nécessaires en vue de leur application, les exécuter et veiller à leur exacte application ;

414 4) Lorsque l'Assemblée législative n'est pas en session, décréter la suspension des droits et garanties constitutionnelles mentionnées à l'alinéa 7) de l'article 121, dans les cas et les limites qui ont été établis par cet alinéa et rendre compte immédiatement à l'Assemblée. Le décret de suspension des garanties équivaut, ipso facto, à la convocation de l'Assemblée en session, laquelle devra se réunir dans les quarante-huit heures suivantes. Si l'Assemblée ne confirme pas la mesure à la majorité des deux-tiers des voix de tous ses membres, les garanties seront considérées rétablies.

$415 \mathrm{Si}$, faute de quorum, l'Assemblée ne peut se réunir, elle le fera le jour suivant, quel que soit le nombre des députés. Dans ce cas, le décret du pouvoir exécutif nécessitera d'être approuvé à la majorité des deux-tiers au moins des présents ;

416 5) Exercer l'initiative en matière législative, et le droit de veto ;

417 6) Maintenir l'ordre et la tranquillité de la Nation, prendre les mesures nécessaires pour la sauvegarde des libertés publiques ;

418 7) Effectuer le recouvrement et le règlement des revenus nationaux conformément à la loi.

419 8) Veiller au bon fonctionnement de l'ensemble des services administratifs ;

420 9) Exécuter et faire exécuter tout ce que décident ou disposent dans l'ordre de leurs compétences les tribunaux et les organismes électoraux à la demande de ceux-ci ;

10) Conclure les accords, les traités publics et les concordats, les promulguer et les exécuter après leur l'approbation par l'Assemblée législative ou par une Assemblée constituante, selon les dispositions de cette Constitution.

Les protocoles résultant des dits traités publics ou conventions internationales qui ne requièrent pas d'approbation législative, entrent en vigueur dès qu'ils ont été promulgués par le pouvoir exécutif.

(Réforme constitutionnelle, loi ${ }^{\circ} 4123$ du 31 mai 1968)

424 11) Communiquer à l'Assemblée législative les informations que celle-ci sollicite dans l'exercice de ses attributions ;

12) Diriger les relations internationales de la République ; 
13) Recevoir les chefs d'Etat ainsi que les représentants diplomatiques, et agréer les consuls des autres nations ;

14) Convoquer l'Assemblée législative en sessions ordinaires et extraordinaires ;

15) Adresser à l'Assemblée législative le projet de budget national au moment et dans les conditions déterminées par cette Constitution;

16) Disposer de la force publique pour préserver l'ordre, la défense et la sécurité du pays ;

17) Délivrer les permis de navigation;

18) Adopter un Règlement adapté au fonctionnement de leurs services, et adopter les autres règlements et les ordonnances nécessaires à la prompte exécution de la loi ;

19) Signer les contrats administratifs non visés à l'alinéa 14) de l'article 121 de cette Constitution; sous réserve de leur approbation par l'Assemblée législative s'ils prévoient des exemptions d'impôts ou de taxes, ou s'ils ont pour objet l'exploitation des services publics, ou des ressources ou des richesses naturelles de l'Etat.

L'approbation législative de ces contrats ne leur confère pas le caractère de loi ni ne les exempte de leur régime juridique et administratif. Les dispositions de cet alinéa ne s'appliquent pas aux emprunts et autres conventions similaires, auxquels se réfère l'alinéa 15) de l'article 121, lesquels sont régis par des règles spéciales.

(Réforme constitutionnelle, loi n 5702 du 5 juin 1975)

20) Accomplir les autres devoirs et exercer les autres attributions que leur confèrent cette Constitution et les lois.

Chapitre III

Les ministres du Gouvernement

Article 141. - Pour la répartition des affaires qui relèvent du pouvoir exécutif, il y aura les ministres que désigne la loi. Un seul ministre pourra être en charge de deux ou plusieurs portefeuilles.

Article 142. - Pour être ministre, il faut :

1) Etre citoyen jouissant de ses droits ;

2) Etre costaricain de naissance, ou par naturalisation avec dix années de résidence dans le pays depuis l'obtention de la nationalité ;

3) Etre d'état laïc ;

4) Avoir 20 ans accomplis.

Article 143. - La fonction de ministre est incompatible avec l'exercice de toute autre charge publique, qu'elle trouve ou non son origine dans l'élection populaire, sauf si des lois spéciales établissant des dispositions spécifiques leur attribuent des fonctions supplémentaires. Sont applicables aux ministres, les règles, les interdictions et les sanctions instituées aux articles 110,111, 112 de cette Constitution, dans la mesure où elles peuvent l'être, aux ministres.

5 Les vice-présidents de la République peuvent être ministres.

Article 144. - Les ministres présentent chaque année à l'Assemblée législative, dans les quinze premiers jours de la première période de sessions ordinaires, un rapport sur les affaires de leur département. 

responsable conjointement avec le président, dans l'exercice des attributions que cette Constitution leur confère ensemble. La responsabilité des actes du Conseil de gouvernement incombera à tous ceux qui ont participé à l'adoption de la décision correspondante.

461 Article 149. - Le Président de la République et le ministre qui ont participé aux actes qui suivent seront aussi conjointement responsables : 
leur décision, ou quand ils font obstacle sous quelque forme que ce soit à l'exercice des fonctions des organismes électoraux ou des municipalités ;

6) Dans tous les autres cas où par action ou par omission, le pouvoir exécutif viole une loi exprès.

Article 150. - La responsabilité du Président de la République, et des ministres pour des faits non délictuels pourra seulement être mise en jeu que lorsque ceux-ci sont en fonction et jusqu'à 4 ans après la cessation de celles-ci.

(Réforme constitutionnelle, loi no 8004 du 22 juin 2000)

Article 151. - Le Président, les vice-présidents de la République ou quiconque assurant la présidence, ne pourront être poursuivis et jugés que si, et après que, en vertu d'une mise en accusation, l'Assemblée législative a déclaré qu'il y a lieu à un procès pénal.

\section{TITRE XI}

\section{LE POUVOIR JUDICIAIRE}

Chapitre unique

Article 152. - Le pouvoir judiciaire est exercé par la Cour suprême de justice et par les autres tribunaux institués par la loi.

Article 153. - En plus des compétences que lui attribue la présente Constitution, il appartient au pouvoir judiciaire de connaître des causes civiles, pénales, commerciales, du travail, et du contentieux administratif ainsi que toutes celles que prévoit la loi, quelles que soient la nature et la qualité des personnes en cause, de prendre à leur sujet des décisions définitives et d'exécuter les jugements qu'il prononce avec l'aide de la force publique si nécessaire.

Article 154. - Le pouvoir judiciaire est soumis à la Constitution et à la loi, et les décisions qu'il prend dans les affaires de sa compétence ne lui imposent pas d'autres responsabilités que celles expressément spécifiées dans la législation.

Article 155. - Aucun tribunal ne pourra se saisir de causes pendantes devant un autre tribunal. Seuls les tribunaux du pouvoir judiciaire pourront demander le règlement d'une affaire ad effectum vivendi.

Article 156. - La Cour suprême de justice est le tribunal supérieur du pouvoir judiciaire et tous les tribunaux, fonctionnaires et les employés de l'ordre judiciaire dépendent d'elle, sans préjudice des dispositions de cette Constitution concernant la fonction publique.

9 Article 157. - La Cour suprême de justice sera formée des magistrats nécessaires pour son bon fonctionnement; ils sont élus par l'Assemblée législative qui constitue les diverses chambres prévues par la loi. Une diminution du nombre de magistrats, quelle qu'en soit l'importance, ne pourra s'effectuer qu'en accord avec les dispositions prévues pour la révision partielle de cette Constitution.

(Réforme constitutionnelle, loi no 1749 du 8 juin 1954)

Article 158. - Les magistrats de la Cour suprême de Justice sont élus pour huit ans et par le vote des deux tiers de la totalité des membres de l'Assemblée Législative. Dans l'exercice de leurs fonctions, ils devront agir avec efficience et pourront être réélus pour une période identique, sauf décision contraire de l'Assemblée législative prise à la majorité des deux-tiers au moins de ses membres.

Les sièges vacants sont pourvus pour une période complète de huit ans. 
(Réforme constitutionnelle, loi $n^{\circ} 8365$ du 15 juillet 2003)

Article 159. - Pour être magistrat il faut :

1) Etre costaricain par la naissance ou par naturalisation, et disposer d'un domicile dans le pays pendant au moins dix années à partir de l'obtention de la carte afférente. Cependant, le Président de la Cour suprême de justice doit être costaricain de naissance ;

2) Etre citoyen en exercice ;

3) Etre d'état laï ;

4) Etre âgé d'au moins trente-cinq ans ;

5) Avoir le titre d'avocat, délivré ou légalement reconnu au Costa Rica, et avoir exercé cette profession pendant au moins dix années, sauf dans le cas des fonctionnaires de l'ordre judiciaire ayant une pratique professionnelle d'au moins cinq années.

(Réforme constitutionnelle, loi no 2026 du 15 juin 1956)

$$
\text { prévue par la loi. }
$$

Article 160. - Ne pourra être élu magistrat celui qui aura un lien de parenté par consanguinité ou par alliance jusqu'au troisième degré compris avec un membre de la Cour suprême de justice.

Article 161. - La qualité de magistrat est incompatible avec toute appartenance aux autres pouvoirs suprêmes.

Article 162. - La Cour suprême de justice nomme son Président parmi les magistrats qui la composent, ainsi que les présidents des diverses chambres, selon des modalités et pour la durée prévues par la loi.

(Réforme constitutionnelle, loi $n^{\circ} 6769$ du 2 juin 1982)

Article 163. - L'élection et le remplacement des juges de la Cour suprême interviennent dans les trente jours civils suivant l'expiration du mandat correspondant ou la date à laquelle la vacance a été annoncée.

(Réforme constitutionnelle, loi $n^{\circ} 8365$ du 15 juillet 2003)

Article 164. - L'Assemblée législative nomme au moins vingt-cinq magistrats suppléants choisis sur une liste de cinquante candidats que lui présente la Cour suprême de justice. Les vacances temporaires des magistrats sont comblées par tirage au sort qu'effectuera la Cour suprême entre les magistrats suppléants. Si un poste de magistrat suppléant devient vacant, l'élection s'opèrera entre l'un des deux candidats que propose la Cour et l'élection a lieu à la première séance ordinaire ou extraordinaire de l'Assemblée législative à compter de la réception par celle-ci de l'avis de vacance. La loi précisera la durée des fonctions, les conditions et les interdictions imposées aux magistrats titulaires, qui ne sont pas applicables aux suppléants.

Article 165. - Les magistrats de la Cour suprême de justice ne pourront être suspendus sans une déclaration selon laquelle il y a lieu de recourir à cette procédure ou pour d'autres raisons définies par la loi dans le chapitre relatif au régime disciplinaire. Dans ce dernier cas, la décision doit être prise par la Cour suprême de justice, au scrutin secret et à la majorité des deux-tiers au moins de ses membres.

Article 166. - En l'absence de dispositions prévues par cette Constitution, la loi désignera la juridiction, le nombre et la durée des fonctions des tribunaux, ainsi que leurs 
attributions, les principes auxquels ils devront se conformer et la manière selon laquelle leur responsabilité pourra être mise en cause.

Article 167. - Pour la discussion et l'approbation des projets de loi relatifs à l'organisation ou au fonctionnement du pouvoir judiciaire, l'Assemblée législative devra consulter la Cour suprême de justice ; pour s'écarter de l'avis rendu par celle-ci, l'Assemblée doit voter le projet à la majorité des deux-tiers de ses membres.

\section{TITRE XII}

\section{LE REGIME MUNICIPAL}

Chapitre unique

Article 168. - Aux fins de l'administration publique le territoire national est divisé en provinces; celles-ci en cantons et les cantons en districts. La loi pourra créer des organisations spéciales.

L'Assemblée législative pourra décréter, en accomplissant les formalités requises pour les révisions partielles de cette Constitution, créer de nouvelles provinces, à condition que le projet correspondant soit approuvé préalablement, par un plébiscite que l'Assemblée fera organiser dans la ou les provinces visées par le démembrement.

La création de nouveaux cantons requiert l'approbation de l'Assemblée législative à la majorité des deux-tiers au moins de ses membres.

Article 169. - L'administration des intérêts et des services locaux dans chaque canton est confiée à une administration municipale, constituée par un organe délibérant composé de conseillers municipaux élus, et d'un administrateur déterminé par la loi.

Article 170. - Les institutions municipales sont autonomes. Dans le cadre du budget ordinaire de la République, sera attribuée à toutes les municipalités du pays une somme correspondant à au moins dix pour cent $(10 \%)$ des recettes estimées pour l'exercice financier concerné.

La loi déterminera les compétences qui seront transférées du Pouvoir Exécutif aux institutions municipales et l'affectation des ressources correspondantes.

510 (Réforme constitutionnelle, loi n 8106 du 3 juin 2001)

511 Article 171. - Les conseillers municipaux sont élus pour quatre ans et doivent obligatoirement remplir leurs fonctions.

512 La loi déterminera le nombre de conseillers et la façon dont ils exercent leurs fonctions. Cependant, les municipalités des cantons centraux des provinces seront composées d'au moins cinq conseillers titulaires et un nombre égal de suppléants.

(Réforme constitutionnelle, loi no 2741 du 12 mai 1971)

514 Article 172. - Chaque district est représenté auprès de la municipalité du canton par un délégué titulaire et un suppléant avec un droit de parole mais sans droit de vote.

Pour l'administration des intérêts et des services dans les districts du canton, dans des cas déterminés, les municipalités pourront créer des conseils municipaux de district, comme organes rattachés à la municipalité correspondante avec une autonomie fonctionnelle propre, qui seront intégrés selon les mêmes procédures d'élection populaire utilisées pour former les municipalités. Une loi spéciale approuvée par les deux-tiers des députés, 
fixera les conditions spéciales dans lesquelles ils peuvent être créés et précisera leur structure, fonctionnement et financement. pour cent des recettes ordinaires prévues pour une année fiscale. Cependant, si cette somme s'avère supérieure à celle qui est nécessaire pour couvrir les besoins essentiels prévus, le département mentionné considèrera la différence comme un revenu exceptionnel, avec un plan de dépense exceptionnelle, afin que l'Assemblée législative prenne les mesures nécessaires. 
531 Pour parvenir à généraliser les assurances sociales et garantir le paiement de la contribution de l'Etat en tant que tel et en tant que patron, des moyens suffisants sont mis à la disposition de la Caisse costaricaine de Sécurité Sociale ; ceux-ci sont calculés de telle façon qu'ils couvrent les besoins actuels et futurs de l'Institution. En cas d'insuffisance de revenus, l'Etat couvrira le déficit et pour cela le pouvoir exécutif devra insérer dans son prochain projet de budget la somme que la Caisse aura jugé nécessaire pour couvrir la totalité des paiements de l'Etat.

Le Pouvoir exécutif préparera, pour l'année économique concernée, les projets de budgets extraordinaires afin d'utiliser les recettes provenant du recours au crédit public ou de toute autre ressource extraordinaire.

(Réforme constitutionnelle, loi $n^{\circ} 2738$ du 12 mai 1961)

Article 178. - Le projet de budget ordinaire sera soumis à l'Assemblée législative par le pouvoir exécutif, au plus tard le premier septembre de chaque année, et la Loi de Budget devra être définitivement adoptée avant le trente novembre de la même année.

Article 179. - L'Assemblée ne pourra augmenter les dépenses budgétaires prévues par le pouvoir exécutif sans établir de nouvelles recettes pour les couvrir, après avis de la Contraloria ${ }^{7}$ générale de la République quant au coût fiscal de celles-ci.

536 Article 180. - Le budget ordinaire et les budgets extraordinaires fixent les limites de l'action des pouvoirs publics en ce qui concerne l'utilisation et la disposition des ressources de l'Etat, et ils ne peuvent être modifiés que par les lois d'origine gouvernementale.

537 Tout projet de modification qui implique d'accroître ou de créer des dépenses devra être soumis aux dispositions de l'article précédent.

538 Cependant, quand l'Assemblée n'est pas en session, le pouvoir exécutif peut modifier l'affectation d'un crédit autorisé, ou ouvrir des crédits additionnels, dans le but exclusif de répondre à des besoins urgents ou imprévus en cas de guerre, de troubles intérieurs ou de calamité publique. Dans de tels cas, la Contraloria ne pourra refuser d'approuver les dépenses ordonnées et le décret correspondant vaudra convocation de l'Assemblée législative en session extraordinaire pour son information.

539 Article 181. - Le pouvoir exécutif transmettra à la Contraloria la liquidation du budget ordinaire et des budgets extraordinaires, au plus tard le premier mars suivant l'expiration de l'année correspondante ; la Contraloria devra la remettre à l'Assemblée, avec son avis, au plus tard le premier mai suivant. L'approbation ou le rejet définitif des comptes appartient à l'Assemblée législative.

Article 182. - Les contrats d'exécution des travaux publics conclu par les autorités de l'Etat, les municipalités et les institutions autonomes, les marchés conclus avec des fonds qui leur sont propres et les ventes ou locations de biens leur appartenant, se feront au moyen de licitations, conformément à la loi pour le montant correspondant.

Chapitre II

La Contraloria Générale de la République ${ }^{8}$

Article 183. - La Contraloria Générale de la République est une institution auxiliaire de l'Assemblée législative pour le contrôle des finances publiques; mais elle jouit d'une indépendance fonctionnelle et administrative totale dans l'accomplissement de ses tâches. 
544 Un contrôleur et un sous-contrôleur sont en charge de la Contraloria. Ces deux fonctionnaires seront nommés par l'Assemblée législative, deux ans après le commencement du mandat présidentiel, pour une durée de huit ans; ils peuvent être réélus indéfiniment, et jouissent des immunités et des prérogatives des membres des pouvoirs suprêmes.

Le contrôleur et le sous-contrôleur sont responsables devant l'Assemblée de l'accomplissement de leurs fonctions ; celle-ci peut les révoquer pour cela à la majorité des deux-tiers au moins de ses membres, si au terme de la procédure sont reconnus leur inaptitude ou le recours à des procédés incorrects.

Article 184. - Les pouvoirs et les obligations de la Contraloria sont :

547 1) Contrôler l'exécution et la liquidation des budgets ordinaires et extraordinaires de la République.

548 Aucun ordre de paiement ne sera émis sur des fonds de l'Etat sans que la dépense concernée ait été visée par la Contraloria ; aucune obligation ne peut être mise à la charge de l'Etat sans avoir obtenu son contreseing.

2) Examiner, approuver ou désapprouver les budgets des municipalités et des institutions autonomes et contrôler leur exécution et leur liquidation.

3) Envoyer annuellement à l'Assemblée législative, au cours de sa première session ordinaire, un mémoire sur ses activités pendant l'année fiscale précédente, comportant des détails sur les activités du Contrôleur et l'exposé des opinions et suggestions que celui-ci considère nécessaires pour le meilleur emploi des fonds publics ;

4) Examiner, contrôler et clore les comptes des institutions de l'Etat et des fonctionnaires ;

5) Et les autres compétences que la Constitution ou les lois lui confèrent.

Chapitre III

Article 185. - Le Trésor National est le centre des opérations de toutes les administrations de revenus nationaux; c'est le seul organisme qui soit légalement compétent pour payer au nom de l'Etat et pour recevoir des sommes d'argent qui à titre de revenus ou pour quelque autre motif, doivent rentrer dans les caisses de la Nation.

Article 186. - Le Trésor est confié à un Trésorier national et à un sous-trésorier. Ces deux fonctionnaires sont indépendants dans l'exercice de leurs fonctions, lesquelles seront réglementées par la loi. Les nominations se font en Conseil de gouvernement, pour une durée de quatre ans, et ces fonctionnaires ne pourront être révoqués que pour une cause juste.

557 Article 187. - Toute dépense à la charge du Trésor national qui ne se rapporte pas aux salaires du personnel permanent de l'administration publique prévus dans le budget, devra être publiée au Journal officiel.

Sont dispensées de la formalité de publication, les dépenses que le Conseil de gouvernement considère, pour des raisons spéciales, comme ne devant pas être publiées. Dans ce cas une information confidentielle et immédiate, sera assurée à destination de l'Assemblée législative et de la Contraloria. 


\section{TITRE XIV}

\section{manipulent des fonds publics, sont obligatoir
devront être évalués, conformément à la loi.}

\section{TITRE XVI}

\section{LES INSTITUTIONS AUTONOMES}

Chapitre unique de leur gestion.

(Réforme constitutionnelle, loi nº 4123 du 31 mai 1968)

Article 189. - Sont des institutions autonomes :

1) Les banques de l'Etat ;

2) Les compagnies d'assurance de l'Etat ; ci.

\section{TITRE XV}

\section{LA FONCTION PUBLIQUE}

Chapitre unique

\section{LE SERMENT CONSTITUTIONNEL}

Chapitre unique l'article 11 de cette Constitution est le suivant : administrative et sont soumises à la loi en matière de gestion. Leurs directeurs répondent

3) Celles que crée la Constitution et les nouveaux organismes qui peuvent être institués par l'Assemblée législative à la majorité des deux-tiers au moins du total de ses membres.

Article 190. - Lors de la discussion et de l'approbation des projets de loi relatifs à une institution autonome, l'Assemblée législative entendra préalablement l'opinion de celle-

Article 191. - Un statut de la fonction publique réglera les relations entre l'Etat et ses employés, afin de garantir l'efficacité de l'administration. publique déterminent, les agents de l'Etat seront nommés en fonction de leur qualification reconnue et ils ne pourront être révoqués que pour un motif valable prévu par la législation du travail; ou, en cas de réduction forcée des services, que ce soit par manque de crédits, ou en vue d'une meilleure organisation des services.

Article 193. - Le Président de la République, les ministres et les fonctionnaires qui ement tenus de déclarer leurs biens, lesquels

Article 194. - Le serment que doivent prêter les fonctionnaires, selon la disposition de

«Jurez-vous devant Dieu et promettez-vous à la Patrie, d'observer et défendre la Constitution et les lois de la République, et d'accomplir fidèlement les devoirs de votre fonction? 
Oui, je le jure.

S'il en est ainsi, que Dieu vous aide, et si non que Dieu et la Patrie vous demandent d'en rendre compte ».

\section{TITRE XVII}

\section{LES REVISIONS DE LA CONSTITUTION}

Chapitre unique

Article 195. - L'Assemblée législative pourra réviser partiellement cette Constitution dans le respect absolu des dispositions suivantes :

1) La proposition de réforme d'un ou de plusieurs articles doit être présentée à l'Assemblée législative pendant une session ordinaire et être signée par dix députés au moins ou par au moins cinq pour cent (5\%), des citoyens inscrits sur les listes électorales ;

(Réforme constitutionnelle, loi nº 8281 du 28 mai 2002)

2) Cette proposition sera lue à trois reprises avec un intervalle de six jours, pour savoir si elle est admise ou non à la discussion ;

3) Dans l'affirmative, elle sera transmise à une commission nommée à la majorité absolue de l'Assemblée, qui se prononce dans un délai de 20 jours ouvrables ;

(Réforme constitutionnelle, loi $n^{\circ} 6053$ du 15 juin 1977)

4) Après présentation du rapport, la discussion a lieu sur les règles établies pour le vote des lois ; la dite réforme devra être approuvée à la majorité des deux-tiers au moins des membres de l'Assemblée ;

5) La décision de procéder à la révision étant prise, l'Assemblée préparera le projet correspondant par une commission, en ce cas, il suffira qu'elle soit approuvée à la majorité absolue ;

6) Le projet mentionné sera transmis au pouvoir exécutif ; celui-ci l'enverra à l'Assemblée avec le message présidentiel, au début de la prochaine législature ordinaire, avec ses observations ou recommandations ;

7) L'Assemblée législative, dans ses premières réunions, devra discuter du projet en trois débats, et si celui-ci est approuvé à la majorité des deux-tiers des membres de l'Assemblée, il formera une partie de la Constitution, et il sera communiqué au pouvoir exécutif pour publication et son observance.

8) Conformément à l'article 105 de la présente Constitution, les réformes constitutionnelles pourront être soumises à référendum après avoir été approuvées au cours d'une législature et avant la suivante, si en décident ainsi les deux-tiers de l'Assemblée législative.

(Réforme constitutionnelle, loi no 8281 du 28 mai 2002)

Article 196. - La révision générale de cette Constitution relève du seul pouvoir d'une Assemblée constituante convoquée à cet effet. La loi qui aura pour objet cette convocation, devra être approuvée à la majorité des deux-tiers au moins des membres de l'Assemblée législative et ne requiert pas la sanction du pouvoir exécutif.

(Réforme constitutionnelle, loi no 4123 du 31 mai 1968) 


\section{TITRE XVIII}

\section{DISPOSITIONS FINALES}

Chapitre unique

Article 197. - Cette Constitution entre en vigueur le huit novembre 1949 et abroge les Constitutions antérieures. L'ordonnancement juridique existant est maintenu en vigueur dans la mesure où il n'est pas modifié ou abrogé par les organes compétents de la puissance publique et où il n'est pas abrogé expressement ou implicitement par la présente Constitution.

\section{DISPOSITIONS TRANSITOIRES}

[Article 10]. - La Chambre qui est créée par l'article 10 comprendra sept magistrats et les suppléants que déterminera la loi, qui seront élus par l'Assemblée législative à la majorité des deux-tiers au moins de ses membres. L’Assemblée législative nommera les membres de la Chambre lors des dix sessions suivant la publication de la présente loi ; deux de celle-ci choisis entre les membres de la Chambre première de la Cour suprême de justice, dont la composition sera ainsi réduite.

Tant que ne sera pas promulguée une loi de la juridiction constitutionnelle, la chambre continuera transitoirement à traiter les questions de sa compétence, ainsi que celles pendantes, conformément aux dispositions en vigueur.

(Réforme constitutionnelle, loi $n^{\circ} 7128$ du 18 août 1989)

[Article 78]. - Tant que ne sera pas promulguée la loi à laquelle se réfère le paragraphe second de l'article 78 de la Constitution, le produit intérieur brut sera déterminé conformément au procédé que le pouvoir exécutif établira par décret.

(Réforme constitutionnelle, loi $n^{\circ} 7676$ du 23 juillet 1997)

[Article 85]. - Pendant la période quinquennale de 1981-1985, la répartition du fonds spécial auquel se réfère le présent article, s'opèrera de la manière suivante : $59 \%$ pour l'Université de Costa Rica; 11,5\% pour l'Institut Technologique de Costa Rica ; 23,5\% pour l'Université Nationale et $6 \%$ pour l'Université d'Etat à Distance.

(Réforme constitutionnelle, loi $n^{\circ} 6580 \mathrm{du} 18$ mai 1981)

[Article 100]. - L'élection des trois nouveaux magistrats suppléants se fera dans les deux mois suivant la promulgation de cette réforme constitutionnelle; dans cet acte la Cour suprême de justice moyennant un tirage au sort, fixera la date et la période de chacun de ces suppléants, de façon à ce qu'elle coïncide avec le terme des périodes des suppléants élus antérieurement à la présente réforme et que à l'avenir il puisse être procédé à l'élection de deux suppléants tous les deux ans.

\section{(Réforme constitutionnelle, loi n 3513 du 24 juin 1965)}

[Article 116]. - L'Assemblée législative issue des élections qui devront se dérouler au mois de novembre mille neuf cent quarante neuf, conformément à la convocation qu'établira le Tribunal Suprême des Elections, s'installera le huit novembre de cette année, et cessera ses fonctions le trente et un octobre mille neuf cent-cinquante-trois. Le Président de la République, les vice-présidents et les députés à l'Assemblée législative issus des élections de mille-neuf-cent-cinquante-trois, dont la date sera opportunément fixée par le Tribunal Suprême des Elections, exerceront leurs charges durant quatre ans et demi, ou: le 
Président et les vice-présidents du 8 novembre de cette année au huit novembre milleneuf-cent-cinquante-huit, et les députés du premier novembre mille neuf cent-cinquantetrois au trente avril mille-neuf-cent cinquante-huit, afin que le mandat présidentiel prenne effet à l'avenir le huit mai, que l'Assemblée s'installe le premier du même mois et que les élections présidentielles et législatives se déroulent en février, le tout la même année.

[Article 132, alinéa 1]. - Les anciens présidents de la République pourront être réélus une fois, conformément aux dispositions de l'article 132 antérieures à la présente réforme.

(Réforme constitutionnelle, loi $n^{\circ} 4349$ du 11 juillet 1969)

611 [Article 141]. - Les ministres qui sont nommés au début du prochain mandat présidentiel auront les compétences déterminées par les lois existantes sur les secrétaires d'Etat, tant qu'il n'est pas légiféré en la matière.

612 [Article 171]. - Les conseillers municipaux élus aux élections de février mille-neuf-centsoixante-deux, exerceront leurs charges du premier juillet mille-neuf-cent-soixante-deux au trente avril mille-neuf-cent-soixante six.

613 (Réforme constitutionnelle, loi $n^{\circ} 2741$ du 12 mai 1961)

614 [Article 177]. - Le pourcentage auquel se réfère l'article 177 pour le budget du pouvoir judiciaire est fixé en une somme qui ne peut être inférieure à trois un quart pour cent pour l'année 1958 ; en une somme qui ne peut être inférieure à quatre pour cent pour l'année 1959 et une somme qui ne peut être inférieure à un pour cent mais pour chacune des années postérieures, le minimum de six pour cent indiqué.

(Réforme constitutionnelle, loi $n^{\circ} 2738$ du 12 mai 1961)

616 [Article 177, paragraphe 3]. - La Caisse costaricaine de sécurité Sociale devra réaliser généraliser les diverses assurances qui sont à sa charge, y compris la protection de la famille dans le régime de maladie et de maternité, dans un délai qui ne peut être supérieur à dix années, à compter de la promulgation de la présente réforme constitutionnelle.

(Réforme constitutionnelle, loi no 2738 du 12 mai 1961)

618 Adoptée dans la salle de l'Assemblée nationale constituante, Palais National, San José le sept novembre mille-neuf-cent-quarante neuf.

\section{NOTES}

1. Texte à jour au $1^{\text {er }}$ octobre 2009 ; langue de travail, l'espagnol. Traduction assurée par Monsieur Justin Daniel, professeur de science politique, Faculté de Droit et d'Economie, directeur du C.R.P.L.C., Université des Antilles et de la Guyane, et Monsieur Jean-Luc Albert, professeur de droit public, Faculté de Droit, Université Jean Moulin, Lyon 3.

1. Palabre.

2. Patronato Nacional de la Infancia.

3. Caja costarricense de seguro social. 
4. Catédra.

5. Juntes electorales.

6. Juntas electorales.

7. L'inspection générale.

8. L'Inspection générale. 